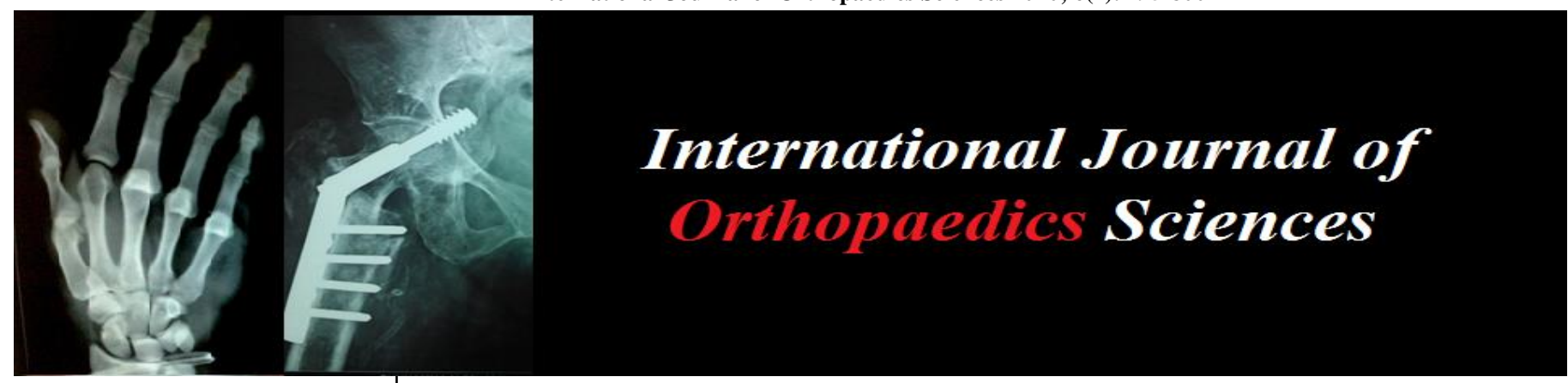

E-ISSN: 2395-1958

P-ISSN: 2706-6630

IJOS 2020; 6(2): 795-800

(C) $2020 \mathrm{IJOS}$

www.orthopaper.com

Received: 12-02-2020

Accepted: 14-03-2020

Maj Suraj Bansal

7 Air Force Hospital,

Cantonment Area, Kanpur,

Uttar Pradesh, India

\section{Dwivedi DD}

Assistant Professor, Govt.

Medical College, Srinagar,

Garhwal, Uttarakhand, India

Kumar S

Professor \& Head, Department of Orthopedics, Sharda

University, Noida, Uttar

Pradesh, India

Puri D

Professor \& Head, Department of Biochemistry, University College of Medical Sciences and Guru Teg Bahadur Hospital, Delhi, India

\section{Mehndiratta M}

Associate Professor, Department of Biochemistry, University College of medical sciences and Guru Teg Bahadur hospital, Delhi, India

Bansal P

Associate Professor, Department of Biochemistry, BPS GMC (W), Sonepat, Haryana, India

\section{Role of bone turnover marker-bone specific alkaline phosphatase evaluation in monitoring healing of tibial shaft fractures managed by closed reduction}

\author{
Maj Suraj Bansal, Dwivedi DD, Kumar S, Puri D, Mehndiratta M and \\ Bansal P
}

DOI: https://doi.org/10.22271/ortho.2020.v6.i2m.2139

\section{Abstract}

Background: Bone is a dynamic tissue and undergoes anabolic and catabolic turnover through action of basic multicellular units of osteoblasts and osteoclasts known as bone metabolic units. Bone-specific alkaline phosphatase (BAP) is a specific marker of osteoblasts and gives a measure of bone forming activity. The tibial fracture is a good model for studying fracture healing in clinical setting because of its frequency of presentation.

Methodology: A study to evaluate the changes in BAP levels during healing of isolated closed tibial shaft fracture was conducted by including 25 healthy adults of age 18 to 45 years. Normal fracture healing time was defined to be 6 months in a tibial shaft fracture. The patients having any systemic condition, disease, and/or drug intake affecting bone turnover were excluded. All the fractures were treated with closed reduction. Follow up was done at every 4 weeks till 6 months of injury to look for clinic-radiological signs of fracture union. Biochemical monitoring was done at time of injury, 4,8 and 12 weeks. BAP was measured using enzyme immunoassay.

Observation: All the fractures united. The baseline value of BAP was $16.9 \pm 6.94 \mathrm{U} / \mathrm{L}$ and it increased by $40.8 \%$ at 4 weeks $(\mathrm{p}=0.005)$, by $71 \%$ at 8 weeks $(\mathrm{p}=0.001)$ and $93.2 \%$ by 12 weeks. BAP and ALP were significantly increased from baseline $(p=0.005$ and $p=0.001)$ in the 21 patients with sticky fracture site as compared to slightly decreased levels in the remaining 4 cases demonstrating mobility in both planes at 4 weeks. BAP and ALP levels at 8 weeks are significantly higher in the 10 patients having visible callus at 8 weeks as compared to the 15 patients having no callus visible at 8 weeks $(\mathrm{p}=0.041$ \& $\mathrm{p}=0.031$ ).

Conclusions: The rise of the marker paralleled the clinic-radiological signs of fracture healing such as absence of mobility at the fracture site and appearance of callus at fracture site at 8 weeks. The bone turnover markers are suggested as a non-invasive aid for monitoring fracture healing complementing the clinic-radiological evaluation.

Keywords: Tibial shaft fractures, bone specific alkaline phosphatase, fracture healing

\section{Introduction}

Clinical examination and radiographic imaging in combination, is considered as the gold standard for monitoring fracture healing. But early detection of a slow healing or a non-healing fracture is not possible with these methods alone ${ }^{[1]}$. An understanding of the changes in the bone turnover markers (BTMs) during fracture repair is of interest since this could lead to the development of new methods of early detection of fractures at risk of delayed or non-union ${ }^{[2]}$. Bone turnover can be demonstrated quantitatively by measuring of a number of molecular markers ${ }^{[3]}$. The 'bone turnover markers' (BTMs) are 1) bone-resorption markers, 2) osteoclast regulatory proteins and 3 ) bone formation markers. Bone-specific alkaline phosphatase (BAP) is a specific marker of osteoblasts and gives a measure of bone forming activity ${ }^{[3]}$. The tibial fracture is a good model for studying fracture healing in clinical setting because of its frequency of presentation ${ }^{[1]}$.

\section{Materials and Methods}

\subsection{Study Design}

A prospective, non-random, observational study to evaluate the changes in serum bone-
Maj Suraj Bansal

7 Air Force Hospital,

Cantonment Area, Kanpur,

Uttar Pradesh, India 
specific alkaline phosphatase levels during healing of isolated tibial shaft fractures was conducted in the department of Orthopaedics and the department of Biochemistry, University College of Medical Sciences and Guru Tegh Bahadur Hospital, a tertiary care hospital in Delhi, India. 25 healthy adults (age 18 to 45 years) with closed fracture tibia were included. Patients with any systemic condition, disease, and/or drug intake affecting bone turnover in any manner were excluded from the study. All the fractures were managed conservatively by closed reduction and plaster cast application. Venous sample were obtained on the day of injury, 4 weeks, 8 weeks and 12 weeks post trauma. The serum was stored at $-40{ }^{\circ} \mathrm{C}$. Samples were analyzed for BAP by Microvue $^{\mathrm{TM}}$ Bone-specific alkaline phosphatase enzyme immunoassay ${ }^{[4,5]}$. Total ALP was measured on autoanalyzer by IFCC recommended kinetic method at 0,4 and 8 weeks. Patients were followed up for 6 months with clinical and radiological examination at every 4 weeks up to 24 weeks. The clinical criteria to assess fracture union were absence of pain or tenderness when weight-bearing, absence of pain or tenderness on palpation or examination, and ability to bear weight and absence of mobility at fracture site in both planes. The plain radiographic criteria are bridging of fracture by callus or bone or trabeculae and bridging of fracture at three cortices and obliteration of fracture line and/or cortical continuity. Delayed union was defined as absence of clinical and radiological signs of healing by 6 months ${ }^{[6]}$.

\subsection{Statistical Analysis}

The elevation in the mean levels of BAP from baseline at 4, 8 and 12 weeks was compared using Wilcoxon Signed Ranks Test. The BAP levels in patients grouped according to clinicradiological parameters such as tenderness at fracture site, weight bearing with or without support and presence of bridging callus on x-ray at different time intervals were compared using Mann Whitney test between groups and by Wilcoxon Signed Ranks Test for serial change in same patients.

\section{Results}

\subsection{Patient Demographics}

The patients comprised of 23 males and 2 females with a mean age of 30.4 years (18 to 45 years). The most common mode of injury was road traffic accident accounting for 15 of the cases. Other modes of injury included household injury (6), fall from height (2) and physical assault (2). 19 patients suffered high energy trauma while 6 patients had a low velocity injury. All these fractures healed within 6 months of injury as evaluated by clinical and radiological methods.

\subsection{BAP \& ALP Measurement}

The mean value of bone specific alkaline phosphatase was $16.9 \pm 6.94 \mathrm{U} / \mathrm{l}$ at baseline, and rose significantly $(\mathrm{p}=0.005)$ by $40.8 \%$ to a $23.8 \pm 10.49 \mathrm{U} / 1$ at 4 weeks. The level reached $28.9 \pm 13.42 \mathrm{U} / 1$ at 8 weeks a $71 \%$ increase from baseline $(\mathrm{p}=0.001)$ and to $32.4 \pm 14.32 \mathrm{U} / \mathrm{l}$ by 12 weeks showing a 93.2\% increase (Table $1 \&$ Figure 1). Similar trends were seen in total alkaline phosphatase levels. (Table 1)

Table 1: Changes in BAP and ALP levels

\begin{tabular}{|c|c|c|c|c|c|c|c|}
\hline & \multicolumn{3}{|c|}{ BAP (U/L) } & & \multicolumn{3}{|c|}{$\operatorname{ALP}(\mathbf{U} / \mathbf{L})$} \\
\hline & Day1 & 4 wks & 8 wks & 12 weeks & Day1 & 4 wks & 8 wks \\
\hline Mean & 16.89 & 23.80 & 28.90 & 32.4 & 75.36 & 96.52 & 103.92 \\
\hline SD & 6.94 & 10.49 & 13.42 & 14.32 & 24.02 & 23.38 & 36.85 \\
\hline$\%$ increase and $\mathrm{p}$ value (from baseline) & & $40.8 \% 0.005$ & $71.0 \% 0.001$ & $93.2 \% 0.001$ & & $28.1 \% 0.001$ & $37.8 \% 0.002$ \\
\hline
\end{tabular}

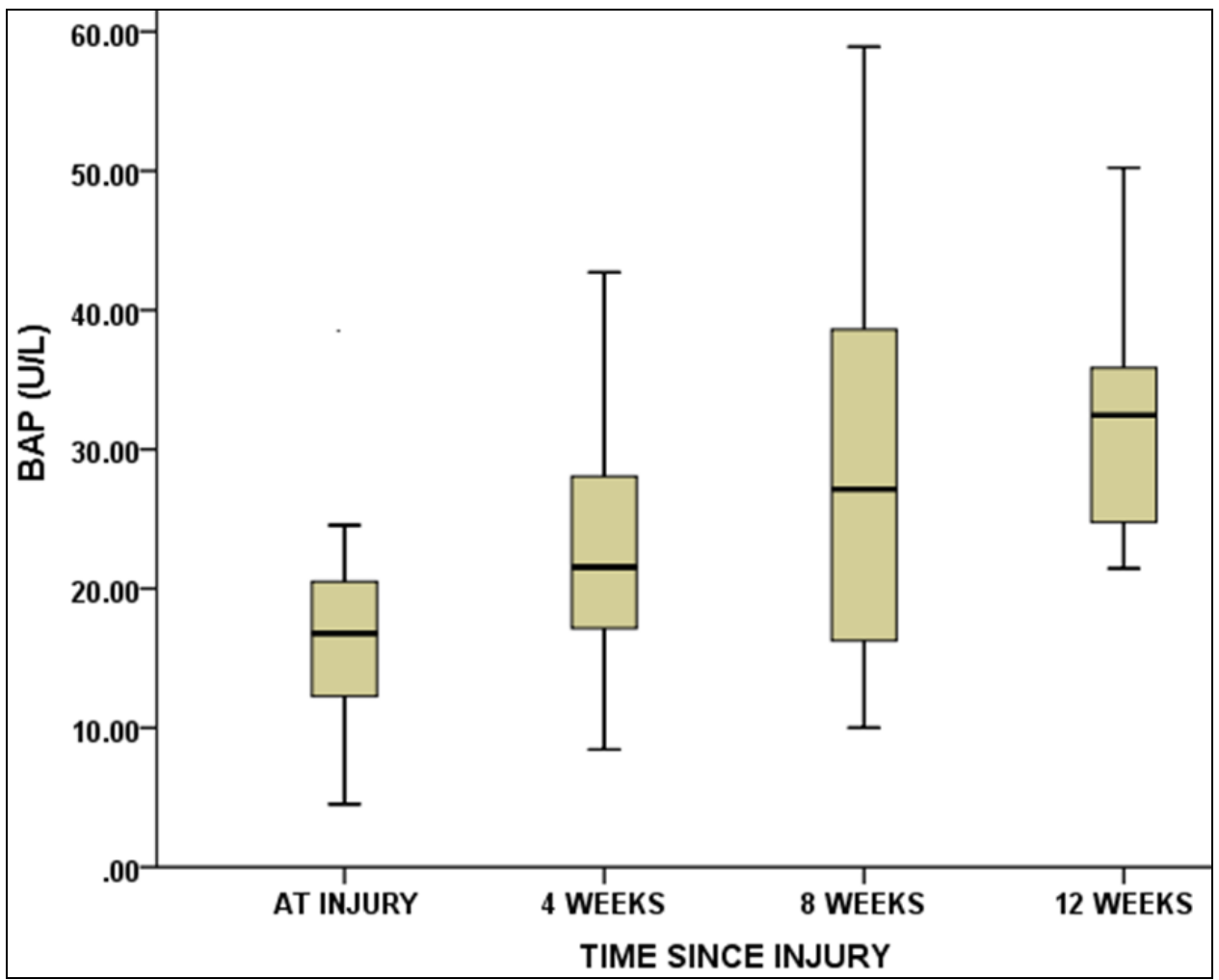

Fig 1: Box plot showing changes in level of bone specific alkaline phosphatase (BAP) at 0, 4, 8 and 12 weeks since injury 
3.3 BAP and ALP levels in Relation to Mobility at Fracture Site (Clinical Assessment)

BAP and ALP were significantly increased from baseline $(\mathrm{p}=$ 0.005 and $\mathrm{p}=0.001)$ in the 21 patients with sticky fracture site (mobility in one plane only). Whereas levels slightly decreased in the remaining 4 cases demonstrating mobility in both planes at 4 weeks (Table 2).

Table 2: Changes in BAP and ALP in relation to mobility at fracture site at 4 weeks

\begin{tabular}{|c|c|c|c|}
\hline \multirow{2}{*}{ Mobility at Fracture Site at 4 weeks } & \multirow{2}{*}{ No. of Cases } & \multicolumn{2}{|c|}{ \% change and p value } \\
\cline { 3 - 4 } & & BAP 0-4 weeks & ALP 0-4 weeks \\
\hline Fracture sticky & 21 & $67.2 \%$ average increase, $\mathrm{p}=0.005$ & $54.2 \%$ average increase, $\mathrm{p}=0.001$ \\
\hline Fracture displaceable & 4 & $32.4 \%$ average decrease, $\mathrm{p}=0.465$ & $10.1 \%$ average decrease, $\mathrm{p}=0.934$ \\
\hline \multicolumn{3}{|c}{} \\
\hline
\end{tabular}

Similarly, at 8 weeks fracture site was non deformable in 22 patients and there was significantly higher rise of both bone specific and total alkaline phosphatase in these patients.
Whereas the rise was not significant in the 3 cases which had displaceable fracture at 8 weeks (Table 3 ).

Table 3: Changes in BAP and ALP in relation to mobility at fracture site at 8 weeks

\begin{tabular}{|c|c|c|c|}
\hline \multirow{2}{*}{ Mobility at fracture Site at 8 weeks } & \multirow{2}{*}{ No. of cases } & BAP 0-8 weeks & \% change and p value \\
\cline { 3 - 4 } & & $107.3 \%$ average increase, $\mathrm{p}=0.001$ & $74.2 \%$ average increase, $\mathrm{p}=0.002$ \\
\hline Fracture not Deformable & 22 & $32.4 \%$ average increase, $\mathrm{p}=0.593$ & $28.7 \%$ average increase, $\mathrm{p}=0.655$ \\
\hline Fracture Deformable & 3 & Wilcoxon Signed Ranks Test (significant at $p<0.05$ ) \\
\hline \multicolumn{2}{|c|}{}
\end{tabular}

\subsection{BAP and ALP Levels in Relation to Callus Formation} at Fracture Site (Radiological Assessment)

BAP and ALP levels at 8 weeks are significantly higher in the 10 patients having visible callus at 8 weeks as compared to the 15 patients having no callus visible at 8 weeks. (Table 4 and Figure 2). Serial radiographs of a patient are shown in figure 3 .

Table 4: Changes in BAP and ALP in relation to call us at fracture site at 8 weeks

\begin{tabular}{|c|c|c|c|}
\hline \multirow{2}{*}{ Level of Marker at 8 weeks } & Callus at 8 weeks & \multirow{2}{*}{ p value } \\
\cline { 2 - 4 } & Absent $(\mathbf{n}=\mathbf{1 5})$ & Present $(\mathbf{n}=\mathbf{1 0})$ & 0.041 \\
\hline BAP & $24.73 \pm 13.26$ & $35.14 \pm 11.60$ & 0.031 \\
\hline ALP & $90.73 \pm 29.10$ & $123.70 \pm 39.76$ & \\
\hline \multicolumn{2}{|c|}{ Mann Whitney test (significant at $p<0.05)$} \\
\hline
\end{tabular}

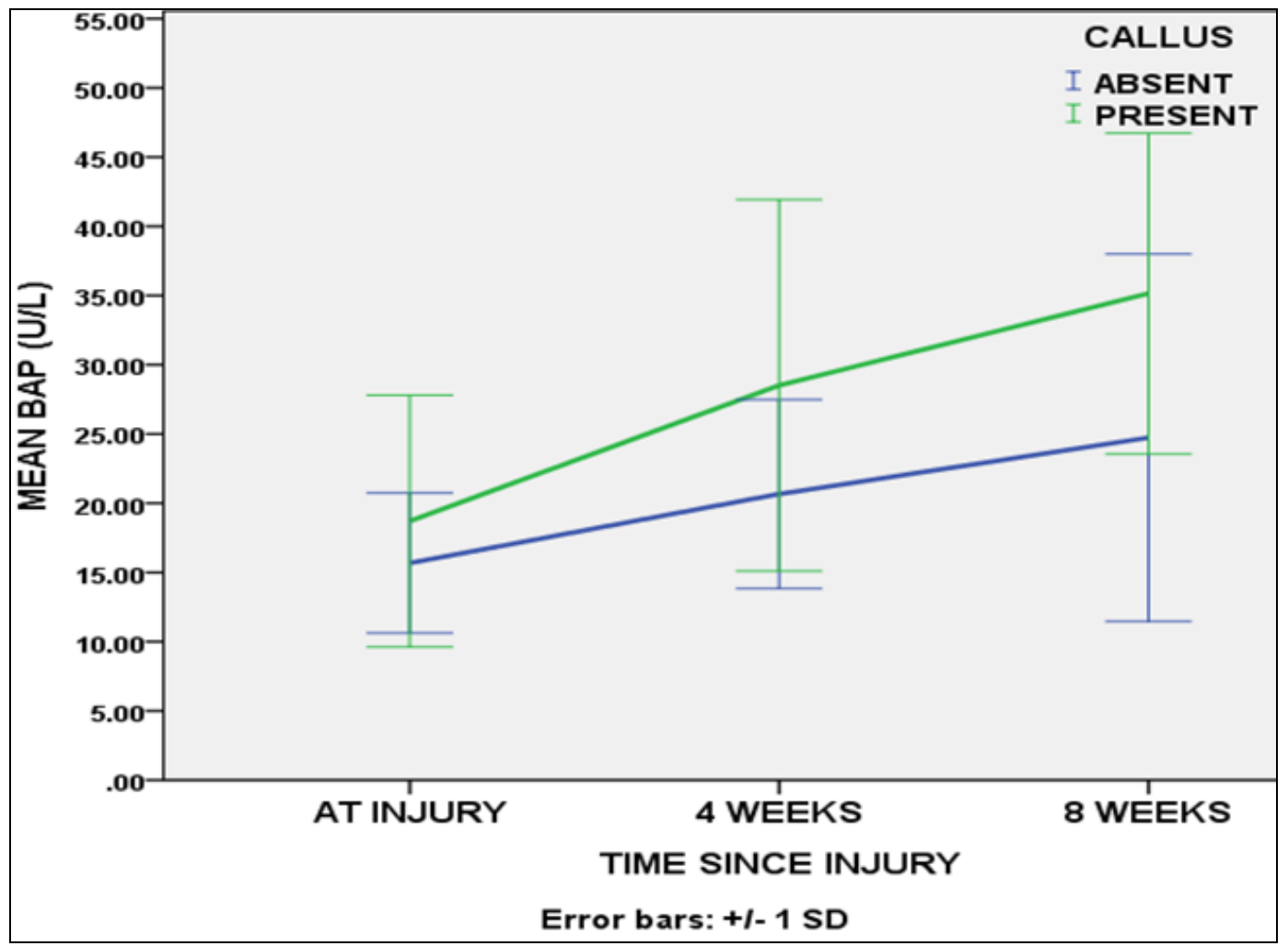

Fig 2: Line diagram showing changes in level of bone specific alkaline phosphatase (BAP) in relation to presence of callus at 8 weeks since injury 


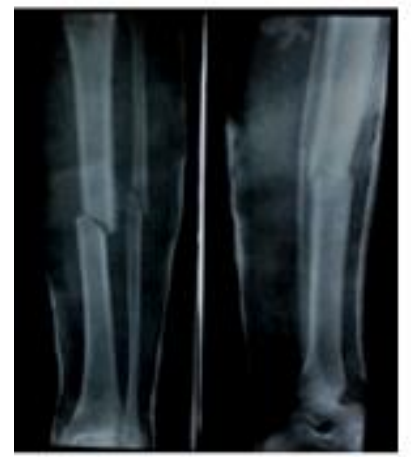

A. DAY $1, B A P=11,9 U / L$ 4 WKS, BAP $=28.3 \mathrm{U} / \mathrm{L}$

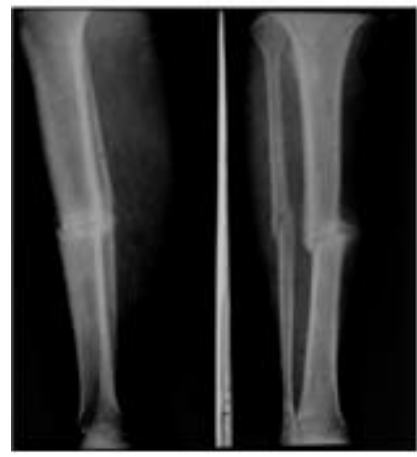

C. 12 WEEKS

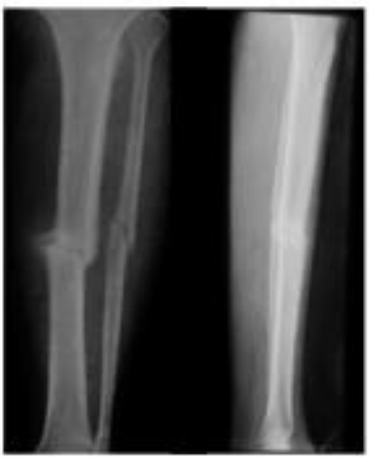

B. 8 WKS, BAP $=52.1 \mathrm{U} / \mathrm{L}$

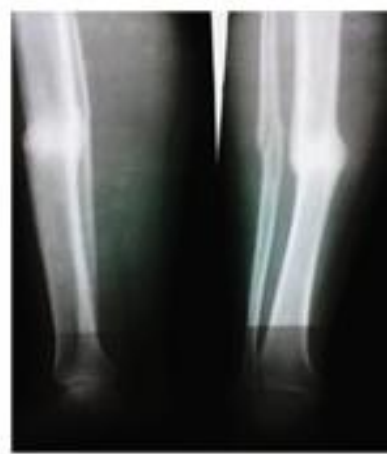

D. 20 WEEKS

Fig 3: Radiological assessment of fracture healing- A. At 4 weeks of injury, B. At 8weeks, C. At 12 weeks, D. At 20 weeks.

\section{Discussion \\ 4.1 Demographics}

The patients comprised of 23 males and 2 females with a mean age of 30.4 years (18 to 45 years). The most common mode of injury was road traffic accident accounting for 15 of the cases. None developed delayed or non-union in the follow up period of 24 weeks.

\subsection{BAP and ALP levels}

Significantly raised levels of bone specific alkaline phosphatase at 4,8 and 12 weeks after fracture signify the increased osteoblastic activity occurring at the fracture site. The rise in levels of the marker was consistent with various previous studies ${ }^{[2,7-12]}$. The time of maximum rise of the level in most cases was from $8-12$ weeks which is also consistent with previous studies ${ }^{\text {[7-12] }}$

\subsection{Correlation with Clinico-Radiological Signs of Fracture Healing}

Correlation between clinical and radiological signs of fracture healing with the levels of bone specific alkaline phosphatase was done. Absence of fracture site mobility at 4 and 8 weeks was associated with significantly higher levels of BAP from baseline in same patients (table 2 and 3). Radiologically callus was visible across the fracture site in 10 of the cases at 8 weeks. The time of appearance of visible callus at the fracture site correlated with the rise in levels of bone specific. These patients had significantly higher BAP levels (table 4 and figure 3). The consolidation of fracture callus by mineralization of soft callus is demonstrated by the raised levels of serum bone specific alkaline phosphatase. BAP levels correlate well with the callus formation at fracture site and thus can be useful tool for monitoring of mineralization and fracture healing. Failure of BAP levels to rise at 4 and 8 weeks can be used as tool to more aggressively treat for impending delayed or non-union.

\subsection{Atypical Trends in Serial BAP Levels}

In four of the cases of our study the level of bone specific alkaline phosphatase decreased from the baseline level at 4 weeks post injury (table 2 and figure 4 ). This could be due to increased osteoclastic activity across the fracture ends compared to osteoblastic activity. The fracture disrupts the blood supply of the bone and the fracture ends undergo a phase of resorption to remove the necrotic bone. This may also signify a delayed osteoblastic response in these patients. However, no significant delay was noted among these patients in time taken for fracture healing clinically or radiologically. The level of bone specific alkaline phosphatase demonstrated a rise between 4 weeks to 8 weeks in these cases showing that osteoblastic activity was present at that time. The fracture callus was consolidating during this period and normal fracture healing was seen (figure 4). In five of the cases of our study the level of bone specific alkaline phosphatase increased at 4 weeks but declined during 4 weeks to 8 weeks period to again rise by 12 weeks. This may be explained by a depressed mineralization of the soft callus by calcium hydroxyapatite crystals to form the hard callus. This can be correlated radiologically with an absence of a visible callus across the fracture site at eight weeks duration. However, clinico-radiologically there was no significant delay in fracture healing in these patients. They were able to bear weight without support at 16 to 20 weeks of the injury and visible callus was present across the fracture site during the subsequent weeks as seen in the skiagram. None of the patients developed delayed or non-union in our study. 


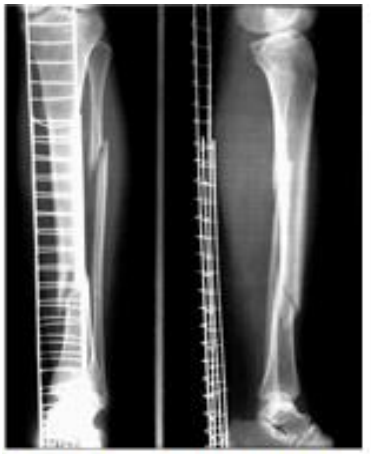

A. DAY 1 , BAP $=16.8 \mathrm{U} / \mathrm{L}$

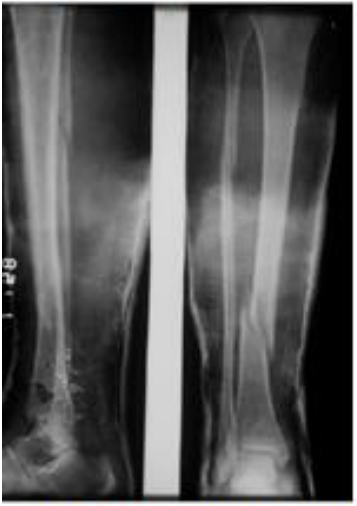

C. 8 WKS, BAP $=13.9 U / L$

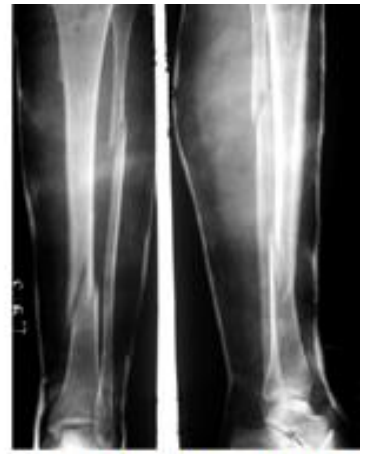

B. 4 WKS, B AP $=8.4 U / \mathrm{L}$

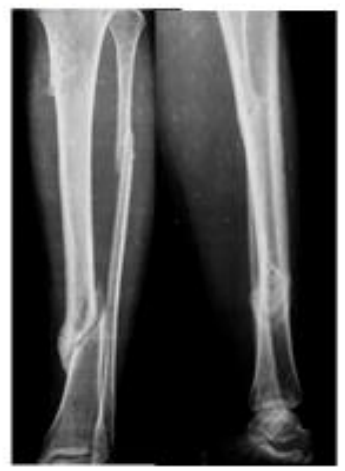

D. 16 WEEKS

Fig 4: Serial skiagram of a case demonstrating the changes of BAP with fracture healing. A. At the time of injury, B. At 4 weeks, C. At 8 weeks, D. At 16 weeks.

\subsection{BAP and ALP in Fracture Healing}

Bone as a living, dynamic tissue undergoes anabolic and catabolic turnover through the action of basic multicellular units of osteoblasts and osteoclast known as the bone metabolic units (BMU). These processes are also activated in fracture healing, metabolic bone diseases and bone neoplasms $[1,2]$. Alkaline phosphatase is a marker elevated in all mineralizing tissues. The function of alkaline phosphatase is not exactly clear, the condition hypophosphatasia, in which the enzyme is lacking is characterized by osteomalacia, suggesting that it has a role in the mineralization of newly formed bone ${ }^{[13]}$. High ALP levels and an increased rate of matrix vesicle production are found in cartilage prior to matrix calcification $[14,15]$. Expression of ALP mRNA is highest 10 to 14 days after fracture in fibrous tissue cells adjacent to newly formed bone, as well as in osteoblasts on the surface of newly formed trabecular bone, but not in the hematoma during the inflammation phase of fracture healing [16]. However, alkaline phosphatase is also found in liver, intestine, and placenta. Therefore we used the skeletal isoform of alkaline phosphatase to monitor fracture healing [17]. Assays of BAP have been developed using electrophoresis, isoelectric focusing, lectin precipitation, and immunoassay techniques. Immunoassay is the method of choice because of high specificity and satisfactory precision ${ }^{[3,18]}$ An increased specificity of monoclonal antibodies for small differences in the molecular structures of antigens forms the basis of further differentiation and specification of ALP isoenzymes among tissues. ALP assays are characterized by minimal diurnal variation ${ }^{[18]}$. Measurement of total alkaline phosphatase is useful when the amount from bone is exceptionally high (such as in Pagets disease of bone and bone forming tumors such as osteosarcoma). Because of the multiple sources of origin and the fact that the bone isoform is usually not increased in osteoporosis and other metabolic bone diseases, total alkaline phosphatase has not enjoyed widespread use as a bone remodeling marker ${ }^{[3]}$. The bone isoform demonstrates a maximum level of activity in the first 6 months of life, falling to a short plateau period between 2 and 9 years rising to a variable degree around the time of puberty before falling to adult levels about the age of 18-20 years ${ }^{[5,19-21]}$. Conditions such as osteomalacia and vitamin D deficiency result in increased levels of ALP despite decreased bone formation. ${ }^{22}$ Evidence has been provided for the stimulating effect of vitamin D3 and its metabolites (1, 25dihydroxycholecalciferol and 24, 25dihydroxycholecalciferol) on ALP expression in osteoblasts in vitro and in vivo ${ }^{[23]}$. A cross-reactivity of approximately $15 \%$ between liver and bone ALP can affect measurements in individuals with high liver ALP ${ }^{[24]}$.

\section{Conclusions}

Thus, we conclude that the levels of serum bone specific alkaline phosphatase correlates with clinico-radiological signs of fracture healing in an isolated tibial diaphyseal fracture. There is a significant rise in the level of the bone formation marker demonstrating an increased osteoblastic activity. The presence of callus at eight weeks also correlates with the level of bone specific alkaline phosphatase suggesting its role in the mineralization of the callus and consolidation of the fracture. The bone turnover markers are suggested as a non-invasive aid for monitoring fracture healing ${ }^{[25]}$. These may help in the assessment of progression of a fracture to union, complementing clinico-radiological methods. However, further studies in this direction can through more light on the complex process of bone healing and the balance between bone formation and resorption to assess the status of BAP levels in patients progressing to non-union or delayed union.

\section{Conflict of Interest and financial disclosures: Nil}




\section{References}

1. Watts NB. Clinical utility of biochemical markers of bone remodeling. Clin Chem. 1999; 45:8(B):1359-68.

2. Cox G, Einhorn TA, Tzioupis C, Giannoudis PV. Boneturnover markers in fracture healing. J Bone Joint Surg [Br]. 2010; 92-B:329-34.

3. Bowles SA, Kurdy N, Davis AM, France MW, Marsh DR. Serum osteocalcin, total and bone-specific alkaline phosphatase following isolated tibial shaft fracture. Ann. Clin. Biochem. 1996; 33:196-200.

4. Gomez B, Ardakani S, Ju J, Jenkins D, Cerelli MJ, Daniloff GY et al. Monoclonal antibody assay for measuring bone-specific alkaline phosphatase activity in serum. Clinical Chemistry. 1995; 41(11):1560-6.

5. Hill CS, Wolfert RL. The preparation of monoclonal antibodies which react preferentially with human bone alkaline phosphatase and not liver alkaline phosphatase. Clin Chim Acta. 1990; 186(2):315-20.

6. Leow JM, Clement ND, Tawonsawatruk T, Simpson CJ, Simpson AH. The radiographic union scale in tibial (RUST) fractures: Reliability of the outcome measure at an independent centre. Bone Joint Res. 2016; 5:116-21.

7. Nyman MT, Paavolainen P, Forsius S, Lamberg-Allardt C. Clinical evaluation of fracture healing by serum osteocalcin and alkaline phosphatase. Ann Chir Gynaecol. 1991; 80(3):289-93.

8. Leung KS, Fung KP, Sher AHL, Li CK, Lee KM. Plasma bone-specific alkaline phosphatase as an indicator of osteoblastic activity. J Bone Joint Surg [Br], 1993, 75B288-92.

9. Ingle BM, Hay SM, Bottjer HM, Eastell R. Changes in bone mass and bone turnover following ankle fracture. Osteoporos Int. 1999; 10:408-15.

10. Ingle BM, Hay SM, Bottjer HM, Eastell R. Changes in bone mass and bone turnover following distal forearm fracture. Osteoporos Int. 1999; 10:399-407.

11. Hermann JM, Klitscher D, Georg T, Frank J, Marzi I, Herrmann W. Different kinetics of bone markers in normal and delayed fracture healing of long bones. Clin Chem. 2002; 48:2263-6.

12. Veitch SW, Findlay SC, Hamer AJ, Blumsohn A, Eastell $\mathrm{R}$, Ingle BM. Changes in bone mass and bone turnover following tibial shaft fracture. Osteoporos Int. 2006; 17:364-72.

13. Whyte MP. Hypophosphatasia and the role of alkaline phosphatase in skeletal mineralization. Endocr Rev. 1994; 15:439-61.

14. Felix R, Herrmann W, Fleisch H. Stimulation of precipitation of calcium phosphate by matrix vesicles. Biochem J. 1978; 170(3):681-90.

15. Anderson HC, Stechschulte DJ Jr, Collins DE, Jacobs $\mathrm{DH}$, Morris DC, Hsu $\mathrm{HH}$ et al. Matrix vesicle biogenesis in vitro by rachitic and normal rat chondrocytes. Am J Pathol. 1990; 136(2):391-8.

16. Chiba S, Okada K, Lee K, Segre GV, Neer RM. Molecular analysis of defect healing in rat diaphyseal bone. J Vet Med Sci. 2001; 63(6):603-8.

17. Oni OO, Mahabir JP, Iqbal SJ, Gregg PJ. Serum osteocalcin and total alkaline phosphatase levels as prognostic indicators in tibial shaft fractures. Injury. 1989; 20(1):37-8.

18. Walton RJ, Preston CJ, Russell RGG, Kanis JA. An estimate of the turnover rate of bone-derived plasma alkaline phosphatase in Paget's disease. Clin Chim Acta. 1975; 63:227-9.
19. Van Hoof VO, Hoylaerts MF, Geryl H, Van Muellem M, Lepourte LG, De Broe ME. Age and sex distribution of alkaline phosphatase isoenzymes by agarose electrophoresis. Clin Chem. 1990; 36:875-8.

20. Whitaker KB, Whitby LG, Moss DW. Activities of bone and liver alkaline phosphatase in serum in health and disease. Clin Chim Acta. 1977; 80:209-20.

21. Crofton PM. Properties of alkaline phosphatase isoenzymes in plasma of pre-term and term neonates. Clin Chem. 1987; 33:1772-82.

22. Crofton PM. Wheat germ lectin affinity electrophoresis for alkaline phosphatase isoenzymes in children: age dependent reference ranges and changes in liver and bone disease. Clin Chem. 1992; 38:663-70.

23. Denney JD, Sherrard DJ, Nelp WB, Chesnut CH, Baylink DJ. Total body calcium and long term calcium balance in chronic renal disease. J Lab Clin Med. 1973; 82(2):22640.

24. Manolagas SC, Spiess YH, Burton DW, Deftos LJ. Mechanism of action of 1, 25-dihydroxyvitamin D3induced stimulation of alkaline phosphatase in cultured osteoblast-like cells. Mol Cell Endocrinol. 1983; 33(1):27-36.

25. Coulibaly MO, Sietsema DL, Burgers TA, Mason J, Williams BO, Jones CB. Recent Advances in the Use of Serological Bone Formation Markers to Monitor Callus Development and Fracture Healing. Crit Rev Eukaryotic Gene Expr. 2010; 20(2):105-27. 